\title{
The Algorithm Research of Indoor Location \\ Based on Ultra Wideband
}

\author{
Jingjing Yang ${ }^{1, \text { a }}$, Yanfei Liu ${ }^{2, b}$ and Xiaogang $\mathrm{Mu}^{3, \mathrm{C}}$ \\ ${ }^{1}$ Xi'an Research Institute of High Technology, Xi'an Shaanxi, 710025, China \\ ${ }^{2}$ Xi'an Research Institute of High Technology, Xi'an Shaanxi, 710025, China \\ ${ }^{3}$ Xi'an Research Institute of High Technology, Xi'an Shaanxi, 710025, China \\ ayangjingjing8405@sina.com, bbbmcu@126.com, ${ }^{\mathrm{C}}$ muxg2001@163.com
}

Keywords: Ultra Wideband;Indoor location.

Abstract. Starting from the research significance of Ultra Wideband positioning, with the basic principles of Ultra Wideband ranging and location as the foundation, this paper introduces four common methods of Ultra Wideband positioning from the two aspects of the basic principle of the locating methods and positioning model. Using three typical decline phenomenon that exists in the transmission process of Ultra Wideband signal as the reference, this paper makes the comparison and analysis of various positioning methods from two aspects of Ultra Wideband technology characteristics and location accuracy.

\section{Introduction}

Recently, with its unique technical advantages more and more attention were paid to ultra-wideband(UWB) technology. Especially application researches about ultra-wideband were raised when ultra-wideband technology was allowed by the federal communications commission (FCC). UWB positioning technology became the potential technology in the wireless location technology with low power consumption, high multipath resolution, low system complexity, especially high positioning accuracy, etc. It has a broad applied prospect in military and civil field. In military field, UWB technology applies extensively because UWB signal has its own special advantages. From the birth of UWB technology, the main application is UWB radar system: weapons that equipped with UWB system could resist enemy radar detection of a band. So the weapons and equipment get stealth performance. Using the high resolution characteristics of narrow pulse that ultra-wideband radar transmitted, the extremely small target could be detected, tracked and monitored in the battlefield. In the civil field, UWB technology uses widely and continues to develop new business to meet the various needs of people's life. For example, in a large warehouse, prison or hospital where people and objects are needed for real-time location tacking, UWB positioning technology becomes the best choice. In disaster relief and emergency situations, UWB positioning technology can greatly help rescue the victims or monitor the risk, etc. Therefore the research of UWB positioning technology has important application value.

\section{Ultra-wideband ranging and location}

Distance refers to compute the distance between two reference node. In the network of reference nodes, a link which leading to a destination node must be established if reference nodes want to get the distance information of destination node. Through this link, the related parameters information of reference node is calculated and the distance between nodes is obtained.

If the transmission signal is given as $\mathrm{s}(\mathrm{t})$, the corresponding signals can be expressed as

$$
\mathrm{r}(t)=h(t) \times s(t)+n(t)
$$

$h(t)$ means impulse response of the channel and $n(t)$ means thermal noise in the expression. If the signal transmit in the ideal channel, the channel impulse response can be expressed as $h(t)=A(D) s(t-\tau(D))$

The received signal can be expressed as $\mathrm{r}(t)=A(D) \delta(t-\tau(D))+n(t)$ 
From the above expression, we can see that distance parameter D can be estimated from attenuation $A(D)$ or time delay $\tau(D)$.

TOA ranging method is based on the signal transmitting time delay between the sender and receiver to calculate the distance between the reference node and destination node. TOA ranging method is one of the most widely used distance measurement method in the field of radar. In a given reference frame of the network, the reference nodes measure the relative distances between other nodes and themselves. In the same way, other each node can measure the relative distances between other nodes and themselves. In these nodes, $\mathrm{k}$ reference nodes can be choosen to form a reference system and to determine their position in the frame of reference according to the geometric calculation method, this is called positioning.

The distance mearsurement and positioning of UWB has some new advantages compared with the traditional ranging positioning technique of continuous wave radio. For example, in some closed indoor and more obstacles environment, many conventional ranging method is difficult to achieve. It becomes a traditional radio gap to jump. With the emergence of UWB technology, it can complete the indoor ranging and location task with its strong penetrating power in the more obstacles environment. According to the principle of TOA ranging method, if the accurate arrival time of pulse signal is obtained, the distance between the source and receiver could be measured accurately. The pulse duration (pulse cycle) of UWB is nversely proportional to the bandwidth of the transmission signal. According to the FCC rules, the bandwidth of the UWB system is up to $7.5 \mathrm{GHz}$, so the time resolution of UWB pulse is up to $133 \mathrm{ps}$, which is equal to the maximum error of pulse transmission time $^{[1]}$. It is only $4 \mathrm{~cm}$ deviation corresponding to the space. If the bandwidth of the UWB signal is $500 \mathrm{MHZ}$, then the corresponding time error limit is up to $2 \mathrm{~ns}$. The distance deviation is $60 \mathrm{~cm}$ corresponding to the space. This means that as long as the attenuation of each signal source of the error of time is not particular obvious, arbitrary UWB signals can complete the positioning task of centimeter level accuracy. This is why the UWB technology is applied to the field of indoor positioning. It is not difficult to know the principle of ranging and location. But it needs to understand its conventional positioning method and to choose the proper positioning method to apply to the UWB indoor positioning, then the UWB technology is used flexible in the field of distance measuring and positioning.

\section{The basic method of Ultra-wide-band positioning}

Generally speaking, the basic methods of positioning are as followed: based on Arrival Time (the Time of arrivals from TOA), based on Arrival Time Difference (the Time Difference of concatenated, TDOA) and based on the Arrival Angle (Angle of arrivals from, AOA) ${ }^{[2]}$. Under the complexity constraints of the established system, each method has its unique advantages and disadvantages. The cost, power, and complexity are the key problems which are often taken into account by its designer in the UWB indoor positioning system. A kind of positioning method with high precision, low computational complexity and easy implementation is inevitably chosen.

\section{TOA}

TOA positioning method measures the signal transmission time between the two or more reference nodes and destination node by technical means. The distance between the reference node and destination node is calculated by physical rules and mathematical formula. Then two or more circles are obtained with the center of the respectively reference nodes position, the radius of the distance which are calculated between the reference node and destination node. The mutual intersection point of the circles is the location of the destination node in theory. Ideally, the TOA positioning method is also known as the Spherical positioning method ${ }^{[3]}$. This method is very effective. The reference model is shown in Fig 1. 


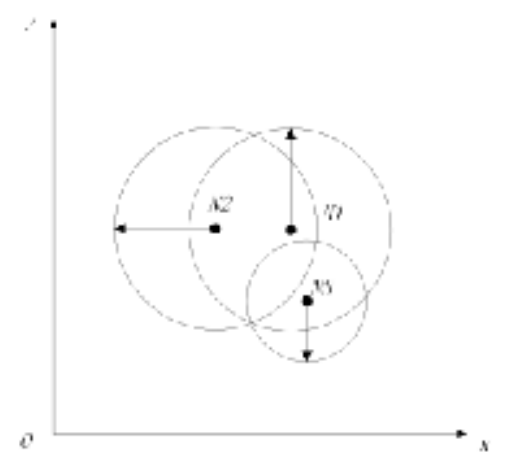

Fig 1 The spherical positioning model of two-dimensional space

The transmission time between the target node and the launch node is set as $\tau$. Then the corresponding distance is $\mathrm{D}=\mathrm{c} \cdot \mathrm{\tau}$, while $\mathrm{c}$ is the speed of light. We imagined that in a three-dimensional space ( $\mathrm{x}, \mathrm{y}, \mathrm{z}$ ), the distance between two nodes $\mathrm{N}_{\mathrm{j}}$ and $\mathrm{N}_{\mathrm{j}}$ is set as $\mathrm{RANG}_{\mathrm{N}_{\mathrm{i}}}\left(\mathrm{N}_{\mathrm{j}}\right)$. Many ball are obtained with $\mathrm{D}_{j i}=R A N G_{N_{i}}\left(N_{i}\right)$ as the radius and the $\mathrm{N}_{j}$ as the center. The location $\left(X_{i}, Y_{i}, Z_{i}\right)$ is identified by the intersect of $\mathrm{k}$ balls which are centered by $\left(N_{1}, N_{2}, \ldots, N_{k}\right)$ with the radius of $\left(D_{1 i} D_{2 i, \ldots} \ldots D_{k i}\right)^{[4]}$. Because in three dimentional space, at least four spheres are required to decide a dot location. Correspondingly four reference nodes are also required. Ideally no additional reference node is not needed to introduce. But in practice, additional auxiliary contacts are introduced to TOA positioning. The accurate destination node is got in three dimentional space through calculating the following equations.

$$
\left\{\begin{array}{c}
\sqrt{\left(X_{1}-X_{i}\right)^{2}+\left(Y_{1}-Y_{i}\right)^{2}+\left(Z_{1}-Z_{i}\right)^{2}} \\
\sqrt{\left(X_{2}-X_{i}\right)^{2}+\left(Y_{2}-Y_{i}\right)^{2}+\left(Z_{2}-Z_{i}\right)^{2}} \\
\ldots \ldots
\end{array}\right\}=\left\{\begin{array}{c}
D_{1 i} \\
D_{2 i} \\
\ldots \ldots \\
D_{k i}
\end{array}\right\}
$$

$\mathrm{K}$ is 4 or more. The accurate location of destination node is also got by the same principle in two-dimensional space.

\section{TDOA}

TDOA location method meaures the arrival time difference between the destination node and two different reference nodes by estimation. The distance between the reference node and destination node is calculated by the time difference. A time difference model is the hyperbolic model with the focus of two reference node, as shown in Fig 2. The position of the destination node is obtained by the intersection of many hyperbolic which is corresponded by the TDOA measurements. So the TDOA location method is often referred as hyperbolic positioning method.

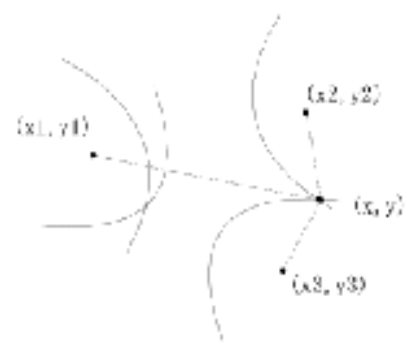

Fig 2 Two-dimensional space hyperbola positioning model

The TOA measurements of the distance between destination node(x,y) and each reference nodes $\mathrm{i}$ and $\mathrm{j}$ are assumed respectively as $\boldsymbol{\tau}_{i}$ and $\mathbf{\tau}_{j}$. Then the reaching time lag of TDOA values determine the corresponding distance which is said as followed.

$$
\mathbf{R}_{i j}=\sqrt{\left(x-x_{i}\right)^{2}-\left(y-y_{i}\right)^{2}}-\sqrt{\left(x-x_{j}\right)^{2}-\left(y-y_{j}\right)^{2}}=c \cdot\left(\tau_{i}-\tau_{j}\right) \quad \mathrm{i}=1,2, \ldots \ldots
$$

$\mathrm{C}$ is the speed of light, $(\mathrm{x}, \mathrm{y})$ is the coordinate of reference node $\mathrm{i},(\mathrm{x}, \mathrm{y})$ is the coordinate of reference node $\mathrm{j}$. TDOA location method only needs to be synchronized between the reference node ${ }^{[5]}$, 
and does not require strict synchronization between the reference node and destination node. Each reference node only needs to have a same clock to keep synchronization. The complexity of the system design is reduced greatly. In the two-dimensional space, not less than 4 reference nodes are required to pinpoint the relative coordinates of the destination node accurately. In the three-dimensional space, 5 reference nodes are required to pinpoint accurately.

AOA

AOA location method is suitable for the stadia LOS conditions. Generally, the incidence angle of which the receiver received the transmit signal through antenna array is measured to obtain the position of the destination node. The interchange of different angle incidence of which is measured by two or more than two antenna reference nodes is the position of the destination node. The positioning model is as shown in Fig 3.

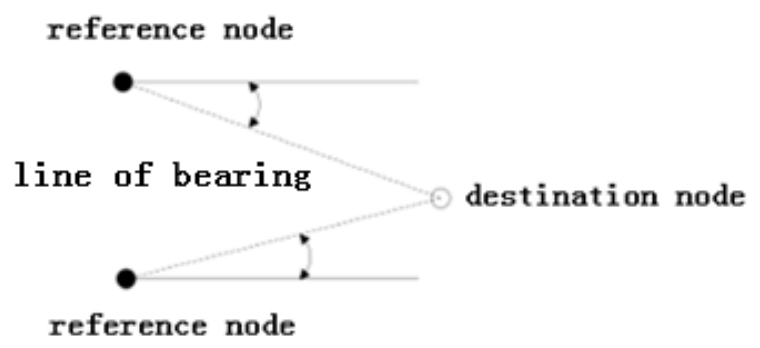

Fig 3 AOA location model of two-dimensional space

In order to measure the incident angle of signal accurately, the antenna array of the receiver must be improved to strength the direction of the antenna array. The common general antenna array are uniform linear array (ULA), uniform circular array (UCA) and cross array. In the algorithm, based on uniform linear array localization method is the most simple, but this method is applicable to the location in the two-dimensional space only ${ }^{[6]}$.

\section{Comparison and analysis of various kinds of positioning method}

The characteristics of the UWB technology are to be put into the above various positioning methods, we can see that carefully: TOA positioning method has a high positioning accuracy in line with the characteristics of high resolution of UWB system. But all the reference nodes of positioning reference frame in the TOA positioning system are required to maintain rigorous and precise synchronization over time, otherwise there will be positioning error. In terms of complexity of the system, TOA positioning system is higher than RSSI positioning system and less than the AOA location system.

TDOA is regarded as a special kind of TOA positioning method based on principle of TOA basis, but the TDOA location system does not need to keep strict time synchronization between reference node and destination node ${ }^{[7]}$, just need to keep time synchronization between each reference node. Thus in terms of complexity of the system, TDOA is slightly less than TOA positioning system.

AOA location method is actually not suitable for UWB positioning. Investigating its reason, mainly because UWB system has wide frequency band. The serious multipath time dispersion is caused by the diffraction effect and reflection effect of the obstacles and objects around in the environment, and the effect in indoor situation is more serious. If we use the maximum likelihood estimation method to multidimensional query the signal of each path, then it will greatly increase the complexity of the system. In addition, the use of array antenna system costs rose sharply, contrary to the principle of system design.

\section{Conclusion}

In addition, comprehensive utilization of various positioning method above is also a kind of positioning strategy. Play to their strengths, to solve the problems arising in the UWB positioning integrally, to improve the positioning accuracy. 


\section{References}

[1] Gezici S. A survey on wireless position estimation[J].Springer Wireless Personal Communications, 2008,44(3).

[2] Wangjinlong, Wang chenggui, Yuchunrong,Xuyitao. The principle and application of Ultra-wide-band (UWB) wireless communication[M].Beijing: Posts and Telecom Press ,2005.

[3] Zhangyong,Qilina.The performance analysis of DS-PAM-UWB RAKE receiver with dense and multipath indoor environment $[[\mathrm{J}]$. Journal of Nanjing University of post and telecommunication,2007,(5).

[4] Sayed A H, Tarighat A, and Khajehnouri N. Network-based Wireless Location[J]. IEEE Signal Processing Magazine, 2005, 22(4).

[5] Jiang Hong, Cao Fucheng, and Ding Rui.Propagator method-based TOA estimation for UWB indoor environment in the presence of correlated fading amplitudes[C].International Conference on Circuits and Systems for Communications 2008.

[6] Wanghongjiang, Weigang,Jifei,Jiangshengming. The research of timing jitter suppression algorithm in UWB communication[J]. Micro computer information,2008, 24.

[7] Wann Chin-der and Hsu Sheng-hsiung. Estimation and analysis of signal arrival time for UWB svstems[C]. IEEE 2004 VTC, 2004, 5. 